JOURNAL OF RESEARCH of the National Bureau of Standards-B. Mathematical Sciences

Vol. 79B, Nos. 1 and 2, January-June 1975

\title{
Linearly Independent Sets of Isotropic Cartesian Tensors of Ranks up to Eight
}

\author{
Elliot A. Kearsley \\ Institute for Materials Research, National Bureau of Standards \\ Washington, D.C. 20234 \\ and \\ Jeffrey T. Fong \\ Institute for Basic Standards, National Bureau of Standards \\ Washington, D.C. 20234
}

\begin{abstract}
This paper contains a complete listing of isotropic Cartesian tensors of ranks up to eight with their associated reduction equations for obtaining linearly independent sets whenever the reduction is called for. In particular, the listing is compiled only for isotropic tensors associated with the rotation group $\mathrm{O}^{+}(3)$ of the three-dimensional underlying vector space. Based on an identity originally due to Capelli (1887), reduction equations for tensors of odd ranks beginning at rank five and even ranks beginning at rank eight are shown to be nontrivial. Significance of the computational result in both pure and applied mathematics is discussed.
\end{abstract}

Key words: Algebra; alternating tensor; Cartesian tensor; Capelli’s identity; group representation; invariant; isotropic tensor; Kronecker delta; orthogonal group; tensor.

\section{Introduction}

With respect to some of the most important groups of linear transformations, Weyl [1] ${ }^{1}$ has written a definitive treatise on the algebraic problem of decomposing a space of tensors of a given rank into its irreducible invariant subspaces. In the course of proving his main results,

A.MS Sublect classification: $15 \mathrm{~A} / 2$

'Figures in brackets indicate the literature references at the end of this paper. 
Weyl employed a powerful formal instrument known as Capelli's identity [1, p. 39]. Based on a theorem proved by Weyl [1, pp. 53-56], and by applying a special form of Capelli's identity, we first demonstrate a nontrivial problem. The problem is that of constructing explicit linearly independent sets of isotropic Cartesian tensors of odd ranks beginning at five and even ranks beginning at eight. Our algebraic results including all reduction equations are then given in full. An application of some of these results in mechanics of deformable media is described in a companion paper [2].

\section{Statement of the Problem}

To facilitate our application in mechanics, we adopt the three-dimensional Euclidean space as the underlying vector space in Cartesian coordinates. With respect to a fixed set of base

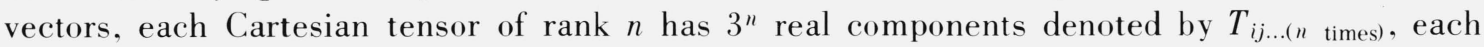
subscript varying from 1 to 3 . Let $A=\left[\alpha_{i j}\right]$ be a rotation matrix satisfying the conditions $\mathbf{A}^{T} \mathbf{A}=1$ and $\operatorname{det} \mathbf{A}=+1$. A Cartesian tensor of rank $n$, with respect to the three-dimensional proper orthogonal group $\mathrm{O}^{+}(3)$, satisfies by definition the following transformation law:

$$
\stackrel{\text { (new) }}{T_{j m q \ldots(n \text { times })}^{(\text {old })}}=T_{i \mathbf{k p} \ldots(n \text { times })} \alpha_{i j} \alpha_{\mathbf{k m}} \alpha_{\mathbf{p q} \ldots(n \text { times })},
$$

where repeated indices denote summation according to the usual convention with each index ranging from 1 to 3 , and the $\alpha$ 's are based on the rotation matrix with which new base vectors are expressed in terms of the old ones.

Within the context of this paper, we define a Cartesian tensor of rank $n$ as isotropic if it satisfies the additional requirement that all components of the tensor remain constant for arbitrary rotations of the coordinate axes. ${ }^{2}$ It is not difficult to show that there is no isotropic Cartesian tensor of rank 1; all isotropic Cartesian tensors of rank 2 are in the form of $\lambda \delta_{i j}, \lambda$ being an arbitrary scalar; and all isotropic Cartesian tensors of rank 3 are scalar multiples of the alternating tensor $\epsilon_{i j k}$ which equals $+1,0,-1$ for cyclic, acyclic, anti-cyclic permutations of the three indices $i, j, k^{3}$ As proved in Weyl [1, pp. 53-56], every isotropic Cartesian tensor of even rank is expressible as a linear combination of products of the Kronecker deltas, $\delta_{i j}, \delta_{k m}$, etc., ${ }^{4}$ and every isotropic Cartesian tensor of odd rank is given by a linear combination of terms formed of products of an appropriate number of Kronecker deltas with an alternating tensor. Such products of Kronecker deltas with or without the alternating tensor $\epsilon_{i j k}$ will be referred to as fundamental isotropic Cartesian tensors (abbrev. FICT). For every rank $n>1$, the total number $N(n)$ of FICT's can be calculated from the following formulas:

$$
\begin{aligned}
& \text { For } n \text { even, } \quad N(n)=\frac{n !}{\left(\frac{n}{2}\right) ! 2^{n / 2}} . \\
& \text { For } n \text { odd, } \quad N(n)=\frac{n !}{3 !\left(\frac{n-3}{2}\right) ! 2^{(n-3) / 2}} .
\end{aligned}
$$

\footnotetext{
${ }^{2}$ Many writers prefer to use the term "hemitropic" or "gyrotropic" for tensors invariant under rotations of the proper orthogonal group

${ }^{3}$ See, e.g., Jeffreys [3, pp. 66-68].

The same result for even rank tensors was given apparently first by Franklin [4] in 1923.
} 
Except for $n=2,3,4$, and 6, Racah [5] showed that $N(n)$ in general exceeds $M(n)$, the total number of linearly independent FICT's. Using the theory of group representation of the threedimensional rotation group, $\mathrm{O}^{+}(3)$, he derived an explicit formula for the number $M(n)$ as follows:

$$
M(n)=\sum_{k=0}^{\left[\frac{n+1}{2}\right]}\left[\left(\begin{array}{c}
n \\
2 k
\end{array}\right)-\frac{1}{2}\left(\begin{array}{c}
n \\
2 k-1
\end{array}\right)\right]\left(\begin{array}{c}
2 k \\
k
\end{array}\right) .
$$

The following table shows that for odd ranks beginning at 5 and even ranks beginning at 8 , there exist linear combinations among FICT's which are identically zero:

TABLF. 1.

\begin{tabular}{l|l|l|l|r|r|r|r|r|r|r}
\hline Rank $n$ & 2 & 3 & 4 & 5 & 6 & 7 & 8 & 9 & 10 & 11 \\
$\begin{array}{l}\text { Number of } \\
\text { Distinct FICT's } \\
\quad N(n)\end{array}$ & 1 & 1 & 3 & 10 & 15 & 105 & 105 & 1,260 & 945 & 17,325 \\
$\begin{array}{l}\text { Number of Linearly } \\
\text { Independent FICT's } \\
M(n)\end{array}$ & 1 & 1 & 3 & 6 & 15 & 36 & 91 & 232 & 603 & 1,585 \\
\hline
\end{tabular}

The problem now is to find these linear combinations, known as reduction equations, for ranks 5, 7, and 8, for use in explicit applications such as those described in [2].

\section{Capelli's Identity}

To obtain the reduction equations for isotropic tensors of odd ranks beginning at five and even ranks beginning at eight, we rely on special cases of a powerful formal instrument known as Capelli's identity. ${ }^{5}$ In particular, we need only to apply the following two identities:

$$
\begin{aligned}
& \left\|\begin{array}{lll}
\delta_{i p} & \delta_{i q} & \delta_{i r} \\
\delta_{j p} & \delta_{j q} & \delta_{j r} \\
\delta_{k p} & \delta_{k q} & \delta_{k r}
\end{array}\right\| \\
& \left\|\begin{array}{llll}
\delta_{i p} & \delta_{i q} & \delta_{i r} & \delta_{i s} \\
\delta_{j p} & \delta_{j q} & \delta_{j r} & \delta_{j s} \\
\delta_{k p} & \delta_{k q} & \delta_{k r} & \delta_{k s} \\
\delta_{m p} & \delta_{m q} & \delta_{m r} & \delta_{m s}
\end{array}\right\|=\epsilon_{i j k} \epsilon_{p q r} .
\end{aligned}
$$

${ }^{5}$ Weyl [1. pp. 39.47] introduced Capelli's identity to prove a fundamental theorem on orthogonal invariants [1. p. 53]. For our purposes here. Weyl's equation (9.1) in [1. p. 53] suffices as the basis for computation. 
Identity (3.1) can be verified directly by expanding the $3 \times 3$ determinant. Identity (3.2) follows from the fact that there are only three possible values of the indices in a three-dimensional space, and since there are four columns on the left hand side, the indices of at least two columns must be equal. ${ }^{6}$ Hence the determinant on the left of (3.2) must vanish. In light of (3.1), consider the determinant of (3.2) expanded in the minors of the first column:

$$
\epsilon_{q r s}\left(\delta_{i p} \epsilon_{j \mathbf{k m}}-\delta_{j p} \epsilon_{i \mathbf{k m}}+\delta_{\mathbf{k} p} \epsilon_{i j m}-\delta_{m p} \epsilon_{i j \mathbf{k}}\right)=0 .
$$

Since the first factor of this equation does not vanish identically, the linear combination of the following four isotropic tensors of rank five must:

$$
\epsilon_{i j k} \delta_{m p}-\epsilon_{j k m} \delta_{i p}+\epsilon_{k m i} \delta_{j p}-\epsilon_{m i j} \delta_{k p}=0 .
$$

Equation (3.4) can be characterized by the index $p$, which is distinguished by occurring in each of the four Kronecker deltas. One can write down a total of five such equations, each characterized by one of the indices $i, j, k, m, p$. A glance at table 1 in the last section, however, reveals that for rank five, six out of ten distinct FICT's are required to form a linearly independent set. Hence one of those five equations based on (3.4) must be dependent. In section 4, we show how a linearly independent set of isotropic tensors of rank five can easily be obtained by using (3.4). A similar technique will be used in section 5 for rank 7 tensors, and in section 6 for rank 8 tensors. A vanishing linear combination such as eq (3.4) is usually referred to as a "null isotropic tensor."

\section{Isotropic Tensors of Ranks 2, 3, 4, 5, and 6}

For completeness, we list here distinct isotropic tensors of ranks 2, 3, 4, and 6, for which no reduction is needed.

\begin{tabular}{|c|c|c|c|c|c|c|}
\hline Rank & \multicolumn{6}{|c|}{ Distinct and linearly independent fundamental isotropic tensors } \\
\hline 2 & \multicolumn{6}{|l|}{$\delta_{i f}$} \\
\hline 3 & \multicolumn{6}{|l|}{$\epsilon_{i j \mathbf{k}}$} \\
\hline 4 & $\delta_{i j} \delta_{\mathbf{k m}}$, & $\delta_{i \mathbf{k}} \delta_{j \mathrm{~m}}$, & $\delta_{i m} \delta$ & & & \\
\hline \multirow[t]{3}{*}{6} & $\delta_{i j} \delta_{k m} \delta_{p q}$ & $\delta_{i j}$ & $\delta_{m \varphi}$ & $\delta_{i j} \delta_{k q} \delta_{m p}$ & $\delta_{i \mathbf{k}} \delta_{j \mathrm{~m}} \delta_{p q}$ & $\delta_{i \mathbf{k}} \delta_{j p} \delta_{m q}$ \\
\hline & $\delta_{i \mathbf{k}} \delta_{j q} \delta_{m p}$ & & ${ }_{j k} \delta_{p q}$ & $\delta_{i m} \delta_{j p} \delta_{k q}$ & $\delta_{i m} \delta_{j q} \delta_{k p}$ & $\delta_{i p} \delta_{j \mathbf{k}} \delta_{m q}$ \\
\hline & $\delta_{i p} \delta_{j m} \delta_{k q}$ & & ${ }_{q} \delta_{k m}$, & $\delta_{i q} \delta_{j \mathbf{k}} \delta_{m p}$ & $\delta_{i q} \delta_{j \mathrm{~m}} \delta_{\mathbf{k p}}$ & $\delta_{i q} \delta_{j p} \delta_{k m}$ \\
\hline
\end{tabular}

TABLE. 2 .

${ }^{6}$ Similar applications of (3.2) in mechanics are in the literature. See, e.g., Rivlin [6]. Spencer and Rivlin [7], etc. 
Independent sets of isotropic tensors of rank five have been studied by Cisotti [8] and Caldonazzo [9]. A particularly simple way of generating such a set is to write eq (3.4) in the following way:

$$
\epsilon_{j k m} \delta_{i p}=\epsilon_{i j k} \delta_{m p}+\epsilon_{i k m} \delta_{j p}-\epsilon_{i j m} \delta_{k p} .
$$

It is immediately clear from this equation, that any fundamental isotropic tensor of rank five in which the index $i$ appears in the Kronecker delta can be expressed as a linear combination of fundamental tensors in which the index $i$ appears in the alternator. A count of the number of the latter fundamental rank-five isotropic tensors reveals that there are six of them, viz:

$$
\epsilon_{i j k} \delta_{m p}, \quad \epsilon_{i j m} \delta_{k p}, \quad \epsilon_{i j p} \delta_{k m}, \quad \epsilon_{i k m} \delta_{j p}, \quad \epsilon_{i k p} \delta_{m j}, \quad \epsilon_{i m p} \delta_{j k}
$$

Since Weyl's result [1] implies that (3.4) or (4.1) exhaust all possible reduction equations for rank-five isotropic tensors, and since none of the six in (4.2) can be so reduced, we conclude that $(4.2)$ is a linearly independent set.

\section{Isotropic Tensors of Rank 7}

The fundamental isotropic tensors of rank seven are products of an alternating tensor and two Kronecker deltas. Table 1 indicates that there are 105 distinct ones for that rank. By the procedure of the last section, it is a simple matter to eliminate all but 45 of these. Thus we need consider only the 45 distinct fundamental tensors generated from $\epsilon_{i j k} \delta_{m p} \delta_{q r}$ by those permutations of the indices which leave the subscript $i$ with the alternator $\epsilon_{i j k}$. Table 1 indicates that a further reduction is necessary since only 36 of these 45 fundamental tensors are linearly independent.

Null isotropic tensors in these 45 fundamental tensors can easily be constructed with the aid of eq (3.4). The product of eq (3.4) with $\delta_{q r}$ can be combined with eq (4.1) in which the indices $q$ and $r$ replace $i$ and $p$, respectively, to give the following equation:

$$
\begin{aligned}
& \epsilon_{i j k} \quad\left(\delta_{m p} \delta_{q r}-\delta_{q p} \delta_{m r}\right)+\epsilon_{i k m}\left(\delta_{j p} \delta_{q r}-\delta_{q p} \delta_{j r}\right) \\
& +\epsilon_{i j m}\left(\delta_{q p} \delta_{k r}-\delta_{k p} \delta_{q r}\right)+\epsilon_{i k q}\left(\delta_{m p} \delta_{j r}-\delta_{j p} \delta_{m r}\right) \\
& +\epsilon_{i m q}\left(\delta_{j p} \delta_{k r}-\delta_{k p} \delta_{j r}\right)+\epsilon_{i j q}\left(\delta_{k p} \delta_{m r}-\delta_{m p} \delta_{k r}\right)=0 .
\end{aligned}
$$

This equation can be characterized by the two indices which appear in the product of Kronecker deltas of every term; i.e., $p$ and $r$. Of the other equations which can be generated by permuting indices, 15 are distinct with a distinct pair of characteristic indices. These equations can be put in the form of a $45 \times 15$ matrix for reduction on a computer.

In order to simplify manipulation of symbols, it is useful to number the 45 fundamental isotropic tensors. This has been done in table 3 , where the symbol $\langle n\rangle$ is assigned to the $n$th tensor of the table. 
$\mathrm{T}_{\mathrm{ABI} . \mathrm{F}}$ 3. Coding of 45 isotropic tensors of rank 7 , of which 36 are independent
$\langle 0\rangle *=\epsilon_{i j k} \delta_{m p} \delta_{q r}$
$\langle 1\rangle *=\epsilon_{i j k} \delta_{m q} \delta_{p r}$
$\langle 2\rangle=\epsilon_{i j \mathbf{k}} \delta_{m r} \delta_{p q}$
$\langle 3\rangle=\epsilon_{i j m} \delta_{k p} \delta_{q r}$
$\langle 4\rangle *=\epsilon_{i j m} \delta_{k q} \delta_{p r}$
$\langle 5\rangle *=\epsilon_{i j m} \delta_{k r} \delta_{p q}$
$\langle 6\rangle=\epsilon_{i j p} \delta_{k m} \delta_{q r}$
$\langle 7\rangle *=\epsilon_{i j p} \delta_{k q} \delta_{m r}$
$\langle 8\rangle=\epsilon_{i j p} \delta_{k r} \delta_{m q}$
$\langle 9\rangle=\epsilon_{i j q} \delta_{k m} \delta_{p r}$
$\langle 10\rangle=\epsilon_{i j q} \delta_{k p} \delta_{m r}$
$\langle 11\rangle=\epsilon_{i j q} \delta_{k r} \delta_{m p}$
$\langle 12\rangle=\epsilon_{i j r} \delta_{\mathbf{k m}} \delta_{\mathbf{p q}}$
$\langle 13\rangle=\epsilon_{i j r} \delta_{\mathbf{k} p} \delta_{\mathbf{m q}}$
$\langle 14\rangle=\epsilon_{i j r} \delta_{k q} \delta_{m p}$
$\langle 15\rangle *=\epsilon_{i \mathbf{k m}} \delta_{j p} \delta_{q r}$
$\langle 16\rangle *=\epsilon_{i \mathbf{k} \mathbf{m}} \delta_{j q} \delta_{p r}$
$\langle 17\rangle=\delta_{\mathbf{i k m}} \delta_{j \mathbf{r}} \delta_{p q}$
$\langle 18\rangle=\epsilon_{i \mathbf{k p}} \delta_{j \mathbf{m}} \delta_{q r}$
$\langle 19\rangle *=\epsilon_{i \mathbf{k} p} \delta_{j q} \delta_{m r}$
$\langle 20\rangle=\epsilon_{\mathrm{ikp}} \delta_{\mathrm{jr}} \delta_{\mathrm{mq}}$
$\langle 21\rangle=\epsilon_{i \mathbf{k q}} \delta_{j \mathbf{m}} \delta_{p r}$
$\langle 22\rangle=\epsilon_{i \mathbf{k q}} \delta_{j p} \delta_{m r}$
$\langle 23\rangle=\epsilon_{\mathbf{i k q}} \delta_{j \mathbf{r}} \delta_{\mathrm{m} \boldsymbol{p}}$
$\langle 24\rangle=\epsilon_{i \mathbf{k r}} \delta_{j m} \delta_{p q}$
$\langle 25\rangle=\epsilon_{i k r} \delta_{j p} \delta_{m q}$
$\langle 26\rangle=\epsilon_{i \mathbf{k r}} \delta_{j q} \delta_{m p}$
$\langle 27\rangle=\epsilon_{i m p} \delta_{j \mathbf{k}} \delta_{q r}$
$\langle 28\rangle *=\epsilon_{i m p} \delta_{j q} \delta_{k r}$
$\langle 29\rangle=\epsilon_{i m p} \delta_{j r} \delta_{k q}$
$\langle 30\rangle=\epsilon_{i m q} \delta_{j \mathbf{k}} \delta_{p r}$
$\langle 31\rangle=\epsilon_{i m q} \delta_{j p} \delta_{k r}$
$\langle 32\rangle=\epsilon_{i m q} \delta_{j r} \delta_{k p}$
$\langle 33\rangle=\epsilon_{i m r} \delta_{j \mathbf{k}} \delta_{p q}$
$\langle 34\rangle=\epsilon_{i m r} \delta_{j p} \delta_{k q}$
$\langle 35\rangle=\epsilon_{i m r} \delta_{j q} \delta_{k p}$
$\langle 36\rangle=\epsilon_{i p q} \delta_{j \mathbf{k}} \delta_{m r}$
$\langle 37\rangle=\epsilon_{i p q} \delta_{j m} \delta_{k r}$
$\langle 38\rangle=\epsilon_{\mathbf{p q q}} \delta_{j \mathbf{r}} \delta_{\mathbf{k m}}$
$\langle 39\rangle=\epsilon_{i p r} \delta_{j \mathbf{k}} \delta_{m q}$
$\langle 40\rangle=\epsilon_{i p r} \delta_{j m} \delta_{k q}$
$\langle 41\rangle=\epsilon_{i p r} \delta_{j q} \delta_{\mathbf{k m}}$
$\langle 42\rangle=\epsilon_{i q r} \delta_{j \mathbf{k}} \delta_{m p}$
$\langle 43\rangle=\epsilon_{i q r} \delta_{j m} \delta_{k p}$
$\langle 44\rangle=\epsilon_{i q r} \delta_{j p} \delta_{\mathbf{k m}}$.

*Marks the nine tensors eliminated by our reduction scheme.

The numerical representation of tensors in table 3 enables us to present the nine reduction equations in a simpler fashion. For example, eq (5.1) becomes

$\langle 0\rangle-\langle 2\rangle-\langle 3\rangle+\langle 5\rangle+\langle 10\rangle-\langle 11\rangle+\langle 15\rangle-\langle 17\rangle-\langle 22\rangle+\langle 23\rangle+\langle 31\rangle-\langle 32\rangle=0$.

The fifteen equations mentioned earlier can all be coded in a form similar to eq (5.2) in order to obtain a $45 \times 15$ matrix for computer solution. This matrix can be reduced by standard procedures to give nine reduction equations expressing nine tensors as linear combinations among the remaining 36 . The nine reduction equations used to eliminate tensors $\langle 0\rangle,\langle 1\rangle,\langle 4\rangle$, $\langle 5\rangle,\langle 7\rangle,\langle 15\rangle,\langle 16\rangle,\langle 19\rangle$ and $\langle 28\rangle$ are shown in table 4 , and the eliminated tensors are marked with asterisks in table 3 . The remaining 36 fundamental tensors of rank 7 form a complete linearly independent set. Such a set is, of course, not unique and entails some arbitrariness in the choice of which tensors are eliminated. 
T ABLE. 4. Reduction equations for isotropic tensors of rank 7

$$
\begin{aligned}
& \langle 0\rangle=2+6-8-10+11-12+13-18+20+22-23+24-25-37+38-43+44 . \\
& \langle 1\rangle=2+9-10-12+13-21+22+24-25-43+44 . \\
& \langle 4\rangle=3-6+8+9-11-13+14+27-29-30+32+37-38+39-40-42+43 . \\
& \langle 5\rangle=3-6+8+12-13+27-29-31+32-33+34+37-38+39-40+43-44 . \\
& \langle 7\rangle=8+10-11-13+14-36+37+39-40-42+43 . \\
& \langle 15\rangle=17+18-20-24+25-27+29+33-34-39+40 . \\
& \langle 16\rangle=17+21-23-24+26-30+32+33-35-42+43 . \\
& \langle 19\rangle=20+22-23-25+26-36+38+39-41-42+44 . \\
& \langle 28\rangle=29+31-32-34+35-37+38+40-41-43+44 .
\end{aligned}
$$

In all nine equations given in table 4 , we have simplified further by dropping all brackets on the right hand side of each equation. The computational result is now complete for rank-seven isotropic tensors.

\section{Isotropic Tensors of Rank 8}

There are 105 fundamental tensors of rank 8, of which only 91 are linearly independent. The null isotropic tensors can be represented by determinants of matrices of Kronecker deltas as was done in eq (3.2). The number of distinct null vectors that can result from permutations of indices of eq (3.2) is 35, that is the number of ways the 8 indices can be divided up into two distinct groups of 4 each. These results can be put into a $105 \times 35$ matrix and reduced in the manner described in the previous section.

The reduction of this large matrix requires considerably more manipulation than does the $45 \times 15$ matrix of the last section. For this reason, it is convenient to have a method of coding the fundamental isotropic tensors by calculation rather than searching an arbitrary list. As it turns out, there is a simple way to do this. The letter indices of the Kronecker deltas are first interchanged with numbers, viz:

$$
(i, j, k, m, p, q, r, s) \rightarrow(7,6,5,4,3,2,1,0) .
$$

Each Kronecker delta then corresponds to a doublet of digits, and these are ordered according to the magnitude of the largest digit (Step 1). The smaller digits of each of the three largest doublets are then collected in order and arrayed from left to right (Step 2). Each of these digits is then reduced by one for each digit to the left of it that is smaller (Step 3). Finally the right hand digit is added to three times the middle digit and 15 times the left hand digit (Step 4). There results a unique number in the range 0 to 104 which characterizes the original fundamental isotropic tensor. The process is illustrated by the following example:

$$
\begin{array}{rllll}
\delta_{i r} \delta_{j q} \delta_{k m} \delta_{p s} \quad \text { becomes } & \text { (Step 1): } & (7,1)(6,2)(5,4)(3,0) & & \\
& \text { (Step 2): } & (7,1)(6,2)(5,4) & \text { or } & (1,2,4) \\
& \text { (Step 3): } & (1,2-1,4-1-1) & \text { or } & (1,1,2) \\
& \text { (Step 4): } & (1 \times 15+1 \times 3+2) & \text { or } & \langle 20\rangle
\end{array}
$$


The inverse coding process is easily constructed. This coding procedure, though mysterious at first glance, relates in a straightforward way with the method of indexing the Kronecker deltas. It is a procedure which can be generalized to arbitrary even ranks of fundamental isotropic tensors. For the rank 8 case, it is convenient to have a dictionary of this coding to use in interpreting results. Table 5 is such a dictionary.

TABLE 5. Dictionary of rank 8 isotropic tensors
$\langle 0\rangle=i$ is $k q m p$
$\langle 1\rangle=$ is jr $k p m q$
$\langle 2\rangle=i$ jr $\mathrm{km} \mathrm{pq}$
$\langle 3\rangle=i$ is $\mathrm{kr} m \mathrm{mp}$
$\langle 4\rangle=i s j q k m r$
$\langle 5\rangle=i$ is $\mathrm{km} \mathrm{pr}$
$\langle 6\rangle=i$ is $k r m q$
$\langle 7\rangle=$ is jp kq $\mathrm{mr}$
$\langle 8\rangle=$ is jp km qr
$\langle 9\rangle=i s j m k r p q$
$\langle 10\rangle=$ is jm kq pr
$\langle 11\rangle=i$ is $j m$ kp qr
$\langle 12\rangle=$ is jk $m r p q$
$\langle 13\rangle=$ is jk mq pr
$\langle 14\rangle=i s j k m p q r$
$\langle 15\rangle=$ ir js kq mp
$\langle 16\rangle=\operatorname{ir~js~kp~mq~}$
$\langle 17\rangle=\operatorname{ir} j s \mathrm{~km} p q$
$\langle 18\rangle=\operatorname{ir~jqks~mp}$
$\langle 19\rangle=i r j q k p m s$
$\langle 20\rangle=i r j q \mathrm{~km} p s$

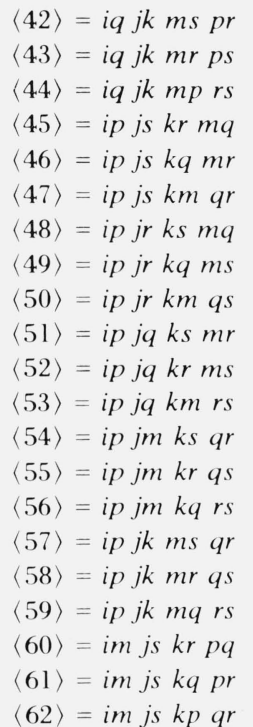

$\langle 42\rangle=i q j k m s p r$

$(21\rangle=\operatorname{ir} j p k s m q$

$\langle 22\rangle=i r j p$ jp $m s$

$\langle 23\rangle=\operatorname{ir} j p \mathrm{~km} q \mathrm{~s}$

$\langle 24\rangle=i r j m k s p q$

$\langle 25\rangle=i r j m k q p s$

$\langle 26\rangle=i r j m k p q s$

$\langle 27\rangle=i r j k m s p q$

$\langle 28\rangle=i r j k m q p s$

$\langle 29\rangle=i r j k m p q s$

$\langle 30\rangle=i q j s \mathrm{kr} m$

$\langle 31\rangle=i q j s k m r$

$\langle 32\rangle=i q j \mathrm{~km} \mathrm{pr}$

$\langle 33\rangle=i q j r k m p$

$\langle 34\rangle=i q j \mathrm{kp} \mathrm{ms}$

$\langle 35\rangle=i q j \mathrm{~km} \mathrm{ps}$

$\langle 36\rangle=i q j p k s \mathrm{mr}$

$\langle 37\rangle=i q j p \mathrm{kr} \mathrm{ms}$

$\langle 38\rangle=i q j p \mathrm{~km} \mathrm{rs}$

$\langle 39\rangle=i q j m k s p r$

$\langle 40\rangle=i q j m k r p s$

$\langle 41\rangle=i q j m k p r s$

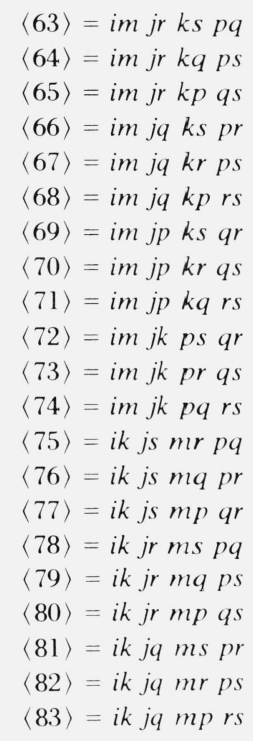

\author{
$\langle 84\rangle=i k j p m s q r$ \\ $\langle 85\rangle=i k j p m r q s$ \\ $\langle 86\rangle=i k j p m q r s$ \\ $\langle 87\rangle=i k j m$ ps qr \\ $\langle 88\rangle=i k j m$ pr qs \\ $\langle 89\rangle=i k j m p q r s$ \\ $\langle 90\rangle=i j k s m r p q$ \\ $\langle 91\rangle=i j k s m q p r$ \\ $\langle 92\rangle=i j k s m p q r$ \\ $\langle 93\rangle=i j k r m s p q$ \\ $\langle 94\rangle=i j k r m q p s$ \\ $\langle 95\rangle=i j k r m p q s$ \\ $\langle 96\rangle=i j k q m s p r$ \\ $\langle 97\rangle=i j k q m r p s$ \\ $\langle 98\rangle=i j k q m p r s$ \\ $\langle 99\rangle=i j k p m s q r$ \\ $\langle 100\rangle=i j k p$ mr qs \\ $\langle 101\rangle=i j k p m q r s$ \\ $\langle 102\rangle=i j k m$ ps qr \\ $\langle 103\rangle=i j \mathrm{~km}$ prqs \\ $\langle 104\rangle=i j \mathrm{~km} p q \mathrm{rs}$
}

The process of reducing the matrix by computer is facilitated by knowing something of the form of the results. Apparently, the reduction equations need never involve coefficients other than 1 and -1 . Whether this is true in general remains, to the best of our knowledge, a conjecture. In light of this, the computer is instructed to abandon the test of any candidate for elimination whenever a coefficient other than \pm 1 appears in the reduced matrix.

The result of the reduction is the elimination of fundamental isotropic tensors $\langle 0\rangle,\langle 1\rangle$, $\langle 12\rangle,\langle 13\rangle,\langle 34\rangle,\langle 35\rangle,\langle 44\rangle,\langle 49\rangle,\langle 58\rangle,\langle 59\rangle,\langle 68\rangle,\langle 75\rangle,\langle 76\rangle,\langle 81\rangle$. The reduction equations are given in table 6 . The remaining 91 tensors are a linearly independent set of isotropic tensors of rank 8. 
TABLF. 6. Reduction equations for rank-eight isotropic tensors

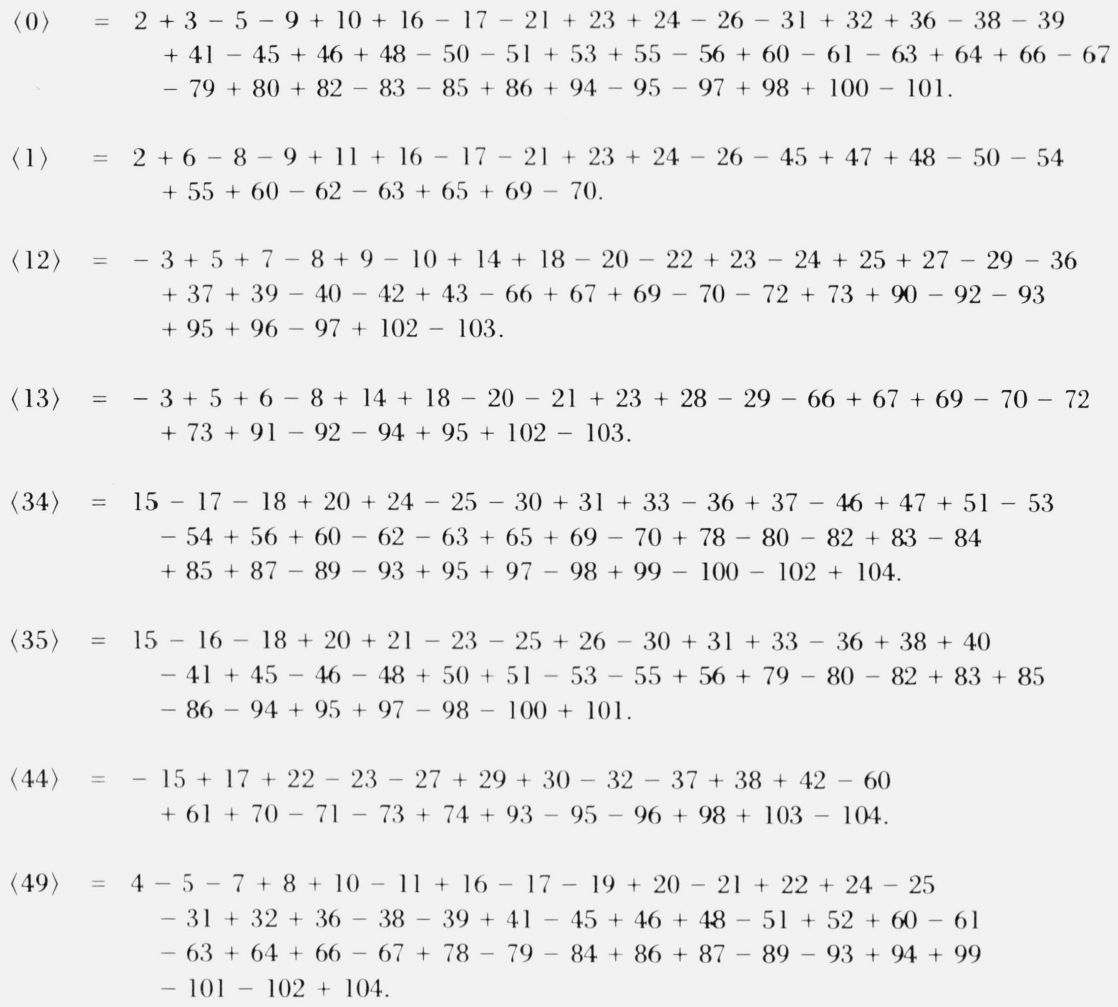

In all 14 equations given in table 6 , we have also simplified further by dropping all brackets on the right hand side of each equation. The computational result is now complete for rank-eight isotropic tensors. 


\section{Significance of Computational Results}

The computational results presented here were motivated by a direct application in mechanics as described in a companion paper [2]. It is probable, however, that further applications in theoretical physics will result in view of the close connection between isotropic tensors and the decomposition of a tensor space. ${ }^{7}$ Since all laws in physics are necessarily tensorial equations, the tool we have developed here would be particularly useful in reducing complicated physical laws into uncoupled irreducible equations.

Two additional comments may be of interest to our readers. On the abstract level, our results may be used to generate examples of mathematically interesting algebras. Tensors invariant to transformations by elements of subgroups of the orthogonal group can be developed also. Such tensors are sometimes called "anisotropic tensors," ${ }^{8}$ and complete sets of them must contain the isotropic tensors developed in this paper. ${ }^{9}$ At the practical level, our results can be used to determine whether two isotropic tensors of equal rank are indeed the same, since the question of "sameness" for two isotropic tensors of equal rank is best settled by expressing them in terms of a linearly independent set. ${ }^{10}$

\section{Acknowledgment}

We wish to thank Professor G. F. Smith of Lehigh University for comments and discussions during the final phase of this work.

\section{References}

[1] Weyl, H., The Classical Groups, Princeton University Press (1939).

[2] Fong, J. T. and Kearsley, E. A., An Application of Higher-Rank Isotropic Tensors to Theory of Non-Simple Materials (in preparation).

[3] Jeffreys, H., Cartesian Tensors, Cambridge University Press (1931).

[4] Franklin, P., Philosophical Magazines, 6 Series 45, 998 (1923).

[5] Racah, G., Determinazione del numero dei tensori isotropi indipendenti di rango n, Rend. d. Reale Accad. Naz. dei Lincei (in Italian), p. 386 (1933).

[6] Rivlin, R. S., J. Rational Mech. Anal. 4, 681 (1955).

[7] Spencer, A. J. M. and Rivlin, R. S., Arch. Rat. Mech. Anal. 2, 309 (1959).

[8] Cisotti, U., Tensori quintupli emisotropi, Rend. d. Reale Accad. Naz. dei Lincei (in Italian) 12 , 195 (1930).

[9] Caldonazzo, B., Osservazione sui tensori quintupli emisotropi, Rend. d. Reale Accad. Naz. dei Lincei (in Italian), Vol. 15, p. 840 (1932).

[10] Fano, U. and Racah, G., Irreducible Tensorial Sets, Academic Press (1959).

[11] Coope, J. A. R., Snider, R. F., and McCourt, F. R., J. Chem. Phys. 43, 2269 (1965).

[12] Coope, J. A. R. and Snider, R. F., J. Math. Phys. 11, 1003 (1970).

[13] Pipkin, A. C. and Rivlin, R. S., Arch. Rat. Mech. Anal. 4, 129 (1959).

[14] Lokhin, V. V. and Sedov, L. I., Nonlinear tensor functions of several tensor arguments, an appendix appearing in A Course in Continuum Mechanics (author; L. I. Sedov; translator: J. R. M. Radok), Volume I, pp. 196-230. Netherlands: Wolters-Noordhoff (1971).

[15] Smith, G. F., The Crystallographic Property Tensors of Orders 1 to 8. Annals of N.Y. Acad. of Sci. 172, 57 (1970).

[16] Smith, G. F., On Isotropic Tensors and Rotation Tensors of Dimension $m$ and order $n$, Tensor 19, 79-88 (1968).

(Paper 79B1\&2-420).

\footnotetext{
${ }^{7}$ See, e.g., Fano and Racah [10], Coope et. al [11, 12].

${ }^{8}$ See, e.g.. Pipkin and Rivlin [13].

9 A convenient listing for the crystal symmetry groups on such invariant tensors up to rank four is given by Lokhin and Sedov [14]. Additional results related to isotropic and anisotropic tensors may also be found in a paper by Smith [15].

${ }^{10}$ This question cannot be answered without the table of reduction equations as given in this paper for ranks up to eight. Smith [16] has shown that. for rank eight tensors, a set of 91 linearly independent elements can be constructed via the "tableau" method. His results offer an alternate approach to the same problem, but contained no explicit reduction equations.
} 